03

\title{
Электролюминесценция одиночных InGaN/GaN микропирамид
}

\author{
(C) A.В. Бабичев ${ }^{1}$, Д.В. Денисов ${ }^{2,3}$, P. Lavenus ${ }^{4}$, G. Jacopin ${ }^{5}$, M. Tchernycheva ${ }^{4}$, F.H. Julien ${ }^{4}$, H. Zhang Zn $^{4,5}$ \\ ${ }^{1}$ Университет ИТМО, \\ 197101 Санкт-Петербург, Россия \\ ${ }^{2}$ Санкт-Петербургский государственный электротехнический университет „ЛЭтИ“ имени В. И. Ульянова (Ленина), \\ 197376 Санкт-Петербург, Россия \\ ${ }^{3}$ Санкт-Петербургский национальный исследовательский Академический университет РАН, \\ 194021 Санкт-Петербург, Россия \\ ${ }^{4}$ Centre for Nanoscience and Nanotechnology (C2N Orsay), CNRS UMR9001, Univ. Paris Sud, Univ. Paris Saclay, \\ 91405 Orsay, France \\ ${ }^{5}$ École Polytechnique Fédérale de Lausanne, \\ $\mathrm{CH}-1015$ Lausanne, Switzerland \\ e-mail: a.babichev@mail.ioffe.ru
}

Поступила в редакцию 17.05.2018 г.

В окончательной редакции 19.10.2018 г.

\begin{abstract}
Представлены результаты отработки технологических режимов формирования и исследования оптических свойств светодиодных микропирамид на основе InGaN/GaN. Структуры сформированы методом металлоорганической газофазной эпитаксии. Светодиодные гетероструктуры на основе одиночных микропирамид демонстрируют электролюминесценцию на длине волны 520-590 nm, которая сдвигается в коротковолновую область с увеличением токовой накачки. Данные источники излучения представляют интерес для формирования точечных источников света высокой интенсивности для биосенсорных применений.
\end{abstract}

DOI: $10.21883 /$ OS.2019.02.47201.130-18

\section{Введение}

Светодиодные гетероструктуры InGaN/GaN на основе нитевидных кристаллов (НК) и микропирамид представляют интерес для ряда практических применений, в том числе для биомедицины (оптогенетики) [1]. Использование геометрии НК [2] или микропирамид [3] обеспечивает рост на неполярных или полуполярных плоскостях, что понижает величину встроенного пьезоэлектрического поля (уменьшает величину пьезоэлектрической поляризации) и плотность прорастающих дислокаций в сравнении с планарными гетероструктурами на основе $\mathrm{GaN}$. Более того, НК/пирамиды перспективны с точки зрения возможности эпитаксии на кремнии [4-6], а также эпитаксии на некристаллических подложках, таких как графен [7-10], перспективны для создания гибких светодиодов на их основе [11-13].

Интерес к формированию $\mathrm{GaN}$ микропирамид, селективно выращенных на поверхности $\mathrm{GaN}$, возник в середине 1990-х годов [14,15]. К настоящему времени продемонстрированы лазеры с оптической накачкой [16], а также светодиоды на основе $\mathrm{GaN}$ микропирамид [17], в том числе гибкие светодиоды $[12,13]$. Наряду с исследованием свойств массива микропирамид [18] проводятся изучения свойств одиночных микропирамид $[3,19,20]$ и НК [21] для оценки их структурного качества [22], исследования оптических свойств [23], исследования механизмов протекания тока [24] и определения уровня легирования в образцах [25].

Одиночные $\mathrm{InGaN} / \mathrm{GaN}$ микропирамиды представляют из себя точечный источник, близкий к идеальному, имеющий высокую яркость в видимом световом диапазоне. Подобные источники представляют интерес для биосенсоров и оптогенетики, например как фотонные зонды для динамического наблюдения биохимических, клеточных процессов или локального возбуждения нейронов [26-29].

В настоящей работе представлены результаты по формированию и исследованию оптических свойств светодиодов на основе одиночных и нескольких InGaN/GaN микропирамид за счет формирования токовой апертуры на поверхности массива микропирамид. Показано, что использование отдельных микропирамид позволяет реализовать точечные сверхкомпактные источники видимого света высокой яркости, представляющие интерес как фотонные зонды высокого разрешения.

\section{Детали эксперимента}

Микропирамиды InGaN/GaN выращены селективно на поверхности $n$-GaN на подложке сапфира через окна в маске, сформированной на основе $\mathrm{Si}_{3} \mathrm{~N}_{4}$ [18]. В качестве методики роста использована металлоорганическая газофазная эпитаксия. Светодиодные пирамиды сформированы в радиальной геометрии ядро-оболочка и включают область ядра, состоящего из $n-\mathrm{GaN}$, покрытого слоем $n$-InGaN с градиентом состава, набор квантовых ям $(\mathrm{InGaN})$, расположенных на полуполярных плоскостях, и оболочку на основе $p-\mathrm{GaN}$. Период расположения микропирамид на подложке составляет $1 \mu \mathrm{m}$, высота 

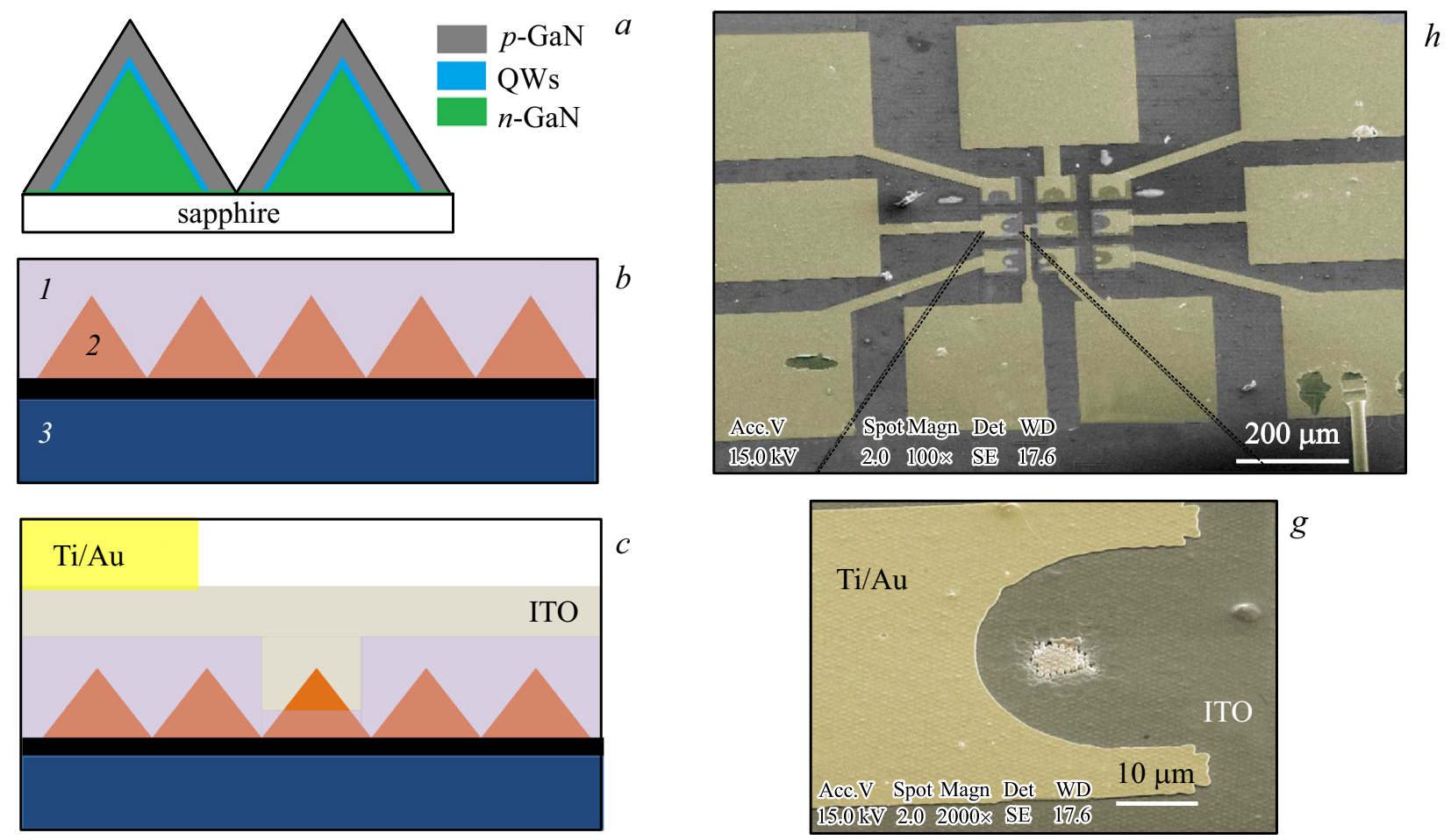

$c$
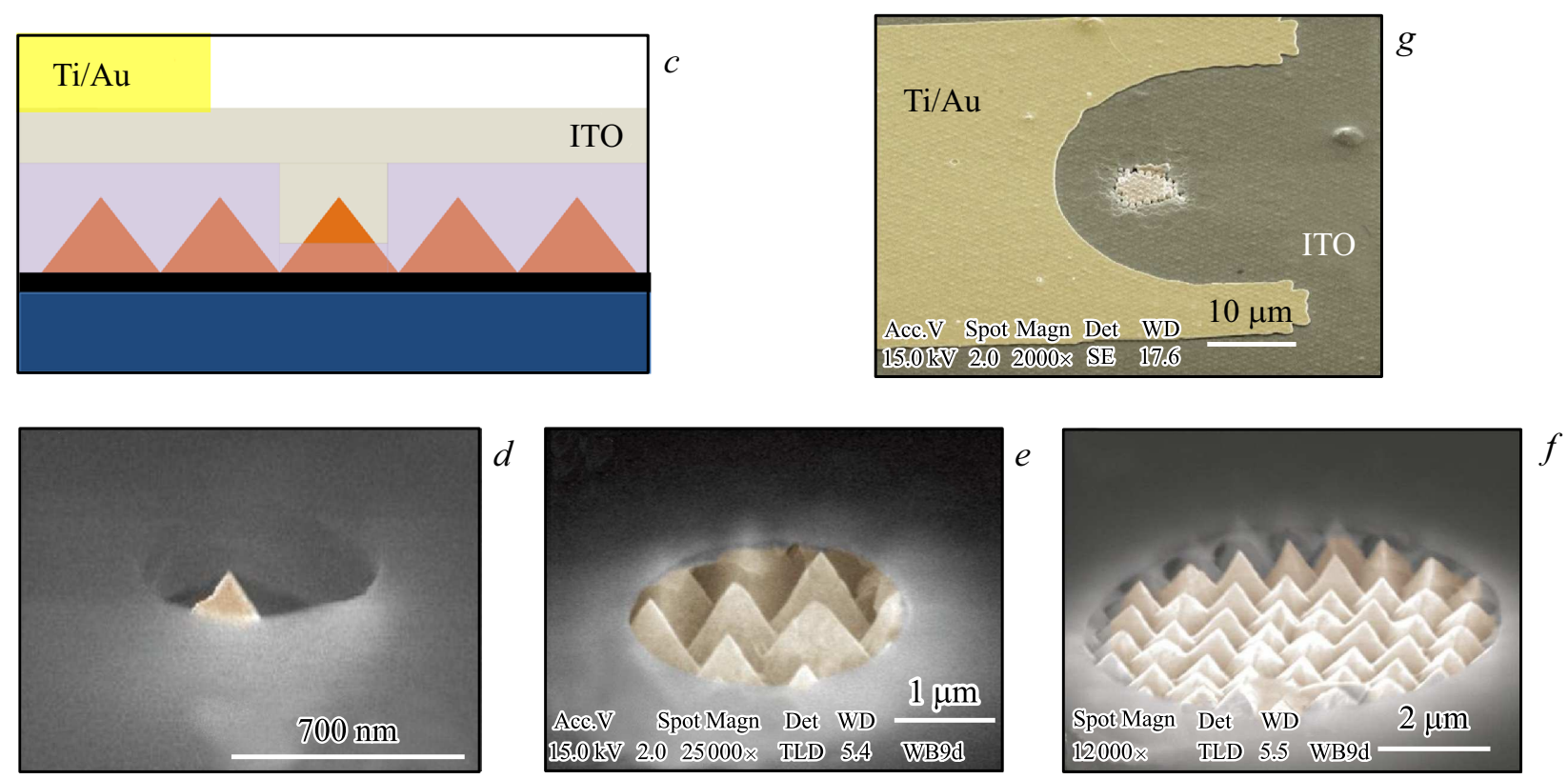

Рис. 1. Схемы и изображения, полученные методом сканирующей электронной микроскопии. $a-$ схематическое изображение скола микропирамид в геометрии ядро-оболочка, $b$ - схематическое изображение скола после центрифугирования HSQ на поверхности массива микропирамид. 1 - HSQ, 2 - микропирамиды, 3 - подложка $n$-GaN на сапфире. $b-$ схематическое изображение скола после формирования окна в маске и травления HSQ для последующего формирования апертуры. $c-$ схематическое изображение скола после нанесения ITO и формирования металлизации Тi/Au. $d-f$ - изображения, полученные методом СЭМ различных апертур (in artificial colors). $h, g$ - изображения, полученные методом СЭМ общего вида сформированных светодиодных гетероструктур.

пирамид $-1.1 \mu \mathrm{m}$. Схематический вид микропирамид в геометрии ядро-оболочка представлен на рис. $1, a$.

Для изучения оптических свойств одиночных микропирамид разработаны шаги процессирования для формирования токовой апертуры. Нанесение нижней металлизации проводилось после вытравливания мезы светодиода методом индуктивно-связанной плазмы до планарного слоя $n$-GaN. Металлизация $n$-контакта формировалась за счет последовательного напыления $\mathrm{Ti}, \mathrm{Al}$, Ti и $\mathrm{Au}$ с толщинами $10,40,30$ и $250 \mathrm{~nm}$ соответственно через маску фоторезиста (взрывная литография). Второй шаг состоял в нанесении методом центрифугирования гидро силсесквиоксана (HSQ) на поверхность микропирамид. Проведена отработка условий нанесения HSQ различной толщины на тестовых структурах, чтобы добиться полного покрытия микропирамид слоем HSQ. Оптимизированная толщина HSQ составила $1.2-1.3 \mu \mathrm{m}$. После выбора оптимальной толщины проводилось нанесение HSQ на исследованные светодиодные микропирамиды (схематически представлено на рис. $1, b$ ). Вторым шагом проводилось центрифугирование негативного электронного резиста ma-N 2400 на поверхности образца и его экспозиция на электронном литографе Raith 150. После проявления ma-N 2400 последний использовался в качестве маски для частичного травления HSQ, формировался профиль окна в диэлектрике. Изображение сканирующей электронной микроскопии $(\mathrm{CЭМ)} \mathrm{для}$ случая открытия окна в диэлектрике с размером, соответствующим основанию микропирамиды, представлено на рис. $1, d$. Также сформированы апертуры бо́льшего 

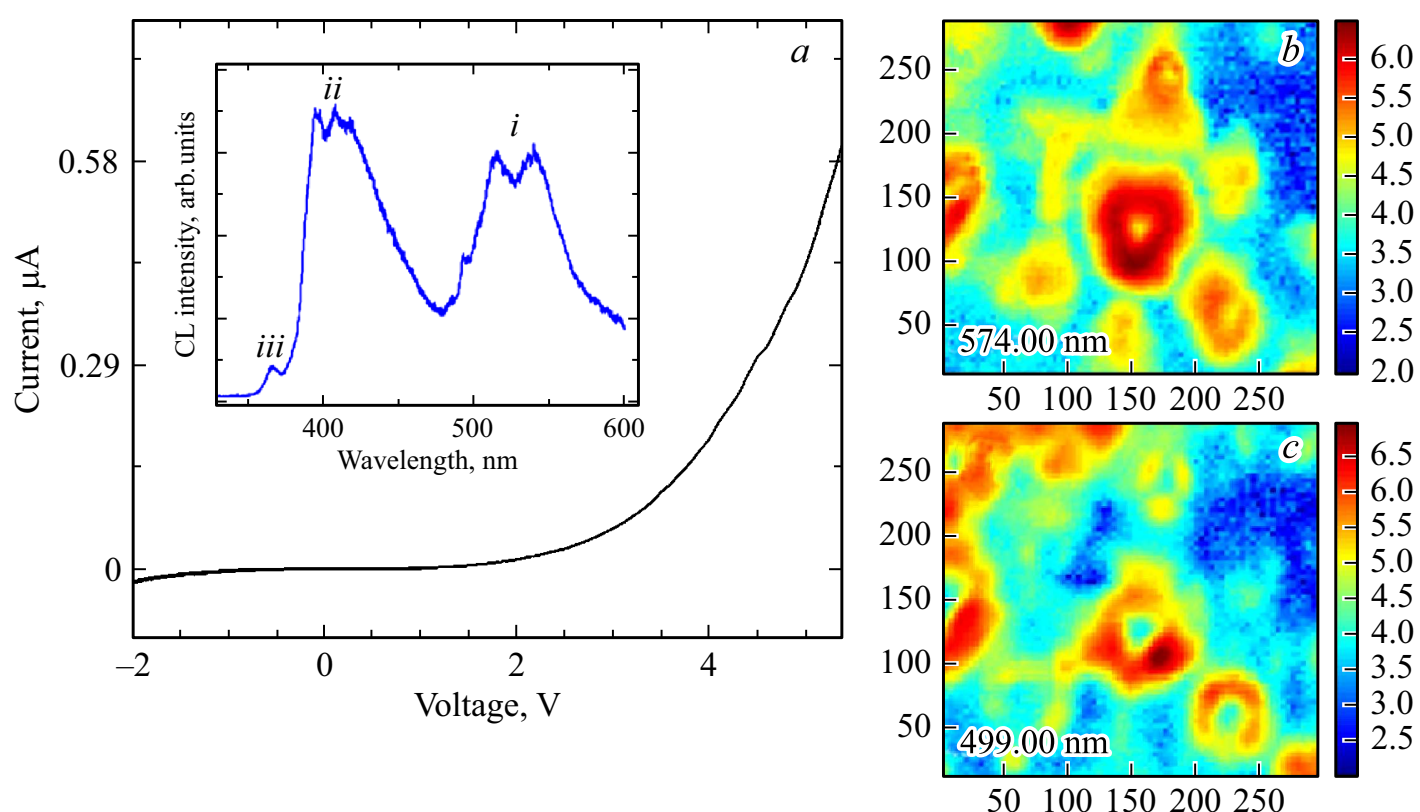

Рис. 2. $a$ - Вольт-амперная характеристика (ВАХ), соответствующая ЭЛ одиночной микропирамиды при диаметре токовой апертуры $10 \mu \mathrm{m}$. Вставка - низкотемпературный спектр КЛ одиночной микропирамиды. $i$ - пик вблизи 500-600 пт, соответствующий квантовой яме InGaN, $i i$ - пик вблизи $400-450 \mathrm{~nm}$, соответствующий $n$-InGaN из области ядра микропирамиды, iii - пик вблизи $370 \mathrm{~nm}$, соответствующий по энергии кванта запрещенной зоне GaN. $b$ - карта КЛ на длине волны излучения $574 \mathrm{~nm} . c$ - карта КЛ на длине волны излучения $499 \mathrm{~nm}$.

диаметра (см. рис. $1, e, f)$. После вскрытия окна в диэлектрике (создания токовой апертуры) произведено нанесение индий-оловянного оксида (ITO) для формирования прозрачного контакта через маску, сформированную методом оптической литографии, схематически представленного на рис. 1,c. Дополнительный шаг оптической литографии применен для формирования верхней металлизации на основе последовательно напыленных слоев $\mathrm{Ti}, \mathrm{Au}$ (методом взрывной литографии) на поверхности ITO для последующей микроразварки. Диаметр апертур варьировался в диапазоне $700 \mathrm{~nm}-10 \mu \mathrm{m}$. Общий вид образцов, полученный методом СЭМ после формирования верхней металлизации, представлен на рис. $1, g, h$.

Спектры катодолюминесценции (КЛ) измерялись при температуре $20 \mathrm{~K}$ и ускоряющем напряжении $5 \mathrm{kV}$ с помощью системы Attolight Alalin Chronos 4027, совмещенной со спектрометром Jobin Yvon (iHr320) и камерой ПЗС (Synapse). Спектры электролюминесценции (ЭЛ) при различных токах накачки измерялись с помощью спектрометра Jobin Yvon с фокальным расстоянием $460 \mathrm{~mm}$ с камерой ПЗС (спектральное разрешение в исследуемом диапазоне составило $\sim 1 \mathrm{~nm})$. Для токовой накачки использован источник-измеритель тока Keithley 2636, подключенный к зондовой станции Janis. Bce измерения проводились при температуре $300 \mathrm{~K}$.

\section{Результаты и их обсуждение}

Продемонстрирована ЭЛ микропирамид с различной токовой апертурой при прямом смещении. Типичная диодная ВАХ одиночной микропирамиды (соответствует случаю светодиода с апертурой в слое HSQ $10 \mu \mathrm{m})$ представлена на рис. 2. Отметим, что, несмотря на большой размер апертуры, ЭЛ наблюдается именно от одиночной микропирамиды в массиве пирамид, заполняющих апертуру. ЭЛ соответствует напряжениям выше $4 \mathrm{~V}$. Несмотря на проведенную отработку условий процессирования апертур в слое HSQ меньшего диаметра (в диапазоне $700 \mathrm{~nm}-10 \mu \mathrm{m})$, ЭЛ для апертур меньшего диаметра наблюдать не удалось. Данный факт может быть связан с флуктуациями контактного сопротивления и с неоднородностями в ростовом процессе, приводящими к неоднородности токовой инжекции в массив микропирамид. Стоит отметить, для мез большой площади $(300 \times 300 \mu \mathrm{m})$, которые были спроцессированы для сравнения, также наблюдалась неоднородность ЭЛ (в среднем ЭЛ наблюдалась только у 5-20\% микропирамид).

Рис. 3, a демонстрирует спектры ЭЛ одиночной микропирамиды, измеренные при различных уровнях накачки. Вставка демонстрирует область ЭЛ для апертуры в слое HSQ диаметром $10 \mu \mathrm{m}$ при приложенном смещении $4.3 \mathrm{~V}$ (ток накачки $0.19 \mu \mathrm{A}$ ) и подтверждает наличие ЭЛ одиночной микропирамиды. В эксперименте продемонстрирована высокая интенсивность ЭЛ, наблюдаемая визуально. Наблюдается пик в желто-зеленой области спектра излучения (соответствует энергии кванта $2.1 \mathrm{eV}$ ), который сдвигается в коротковолновую область с увеличением уровня накачки (до энергии $2.36 \mathrm{eV}$ ). Проведены дополнительные исследования низкотемпе- 

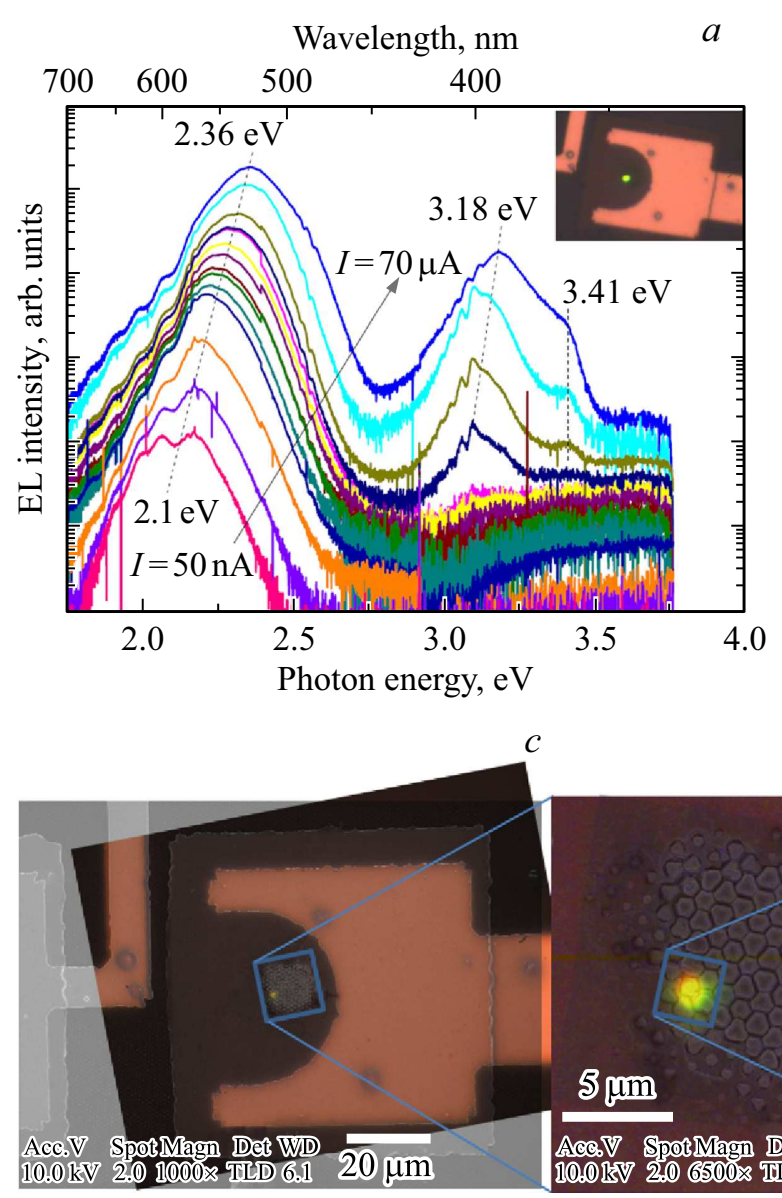
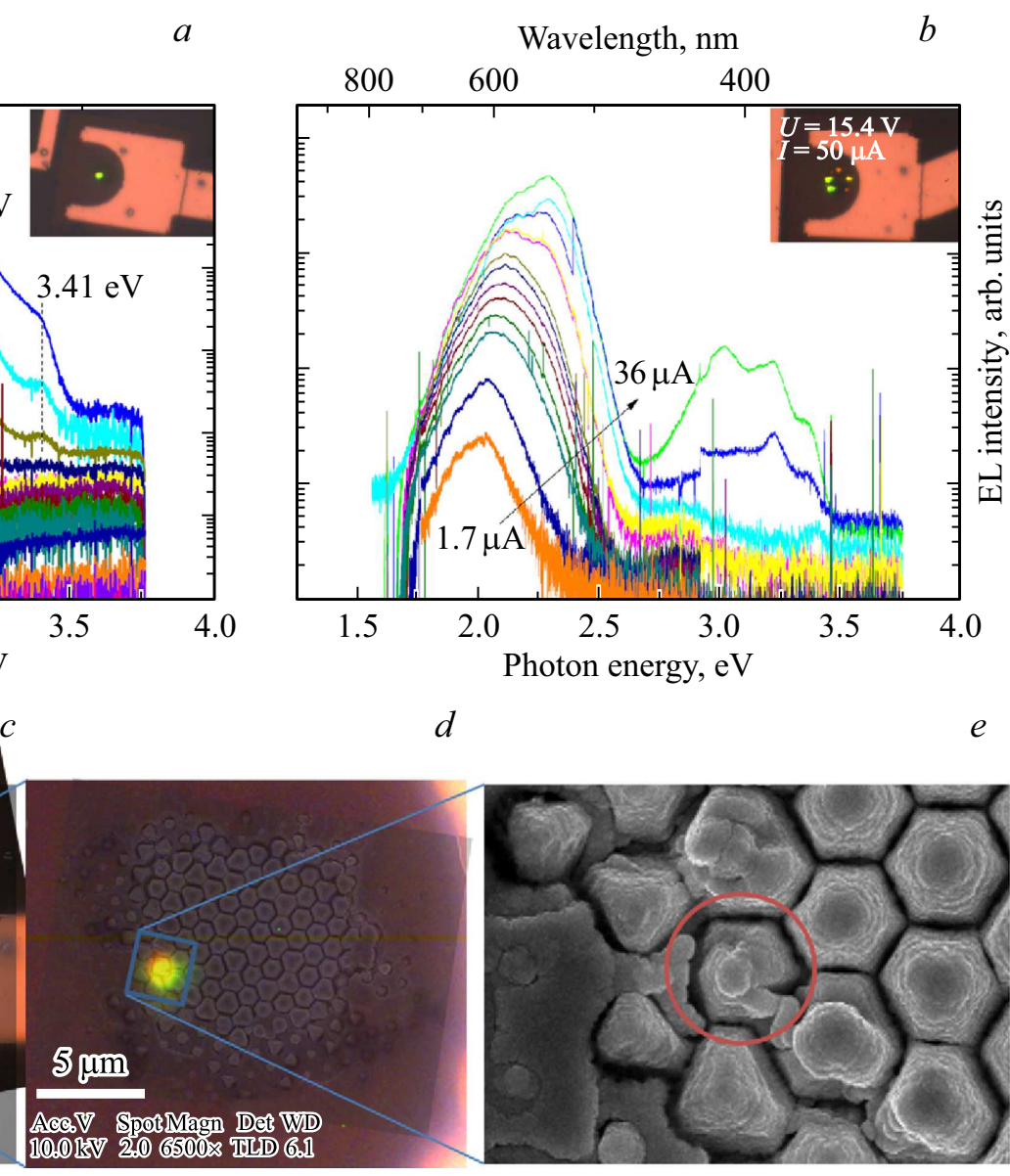

$e$

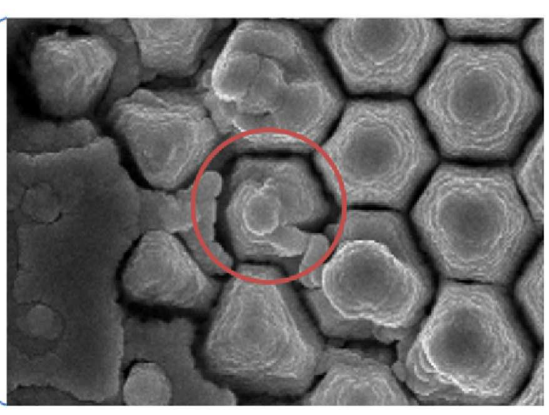

Рис. 3. $a-$ спектры ЭЛ одиночной микропирамиды при различной токовой накачке (в диапазоне $50 \mathrm{nA}-70 \mu \mathrm{A}$, направление увеличения тока накачки отмечено стрелкой). Вставка демонстрирует изображение ЭЛ мезы от одиночной микропирамиды. $b-$ спектр ЭЛ от 5 микропирамид при различной токовой накачке (в диапазоне $1.7-36 \mu \mathrm{A}$, направление увеличения тока накачки отмечено стрелкой). Вставка демонстрирует изображение ЭЛ 5 микропирамид. $c$ - изображения мезы (фотография карты ЭЛ от одиночной микропирамиды (увеличенное изображение, представленное на вставке рисунка $a$ ), наложенная поверх снимка СЭМ изображения). $d-$ увеличенные СЭМ изображение, представленное на $c$ ) с наложенной картой ЭЛ. $e-$ увеличенное СЭМ изображение, представленное на $d$ ).

ратурной КЛ неспроцессированных микропирамид для объяснения изменения спектра ЭЛ с увеличением уровня накачки. Ранее (см. работу [30]) показана зависимость длины волны излучения в спектрах КЛ от положения на грани микропирамид, что соотносится с результатами, представленными на картах КЛ на рис. 2, $b-c$. Вблизи вершины наблюдается область с высоким содержанием индия, дающая длинноволновый вклад в спектре КЛ (рис. $2, b$ ). В свою очередь, вблизи основания микропирамиды квантовая яма имеет меньший состав по индию, что приводит к сдвигу пика спектра КЛ в коротковолновую область (рис. 2,c). Спектр КЛ при возбуждении всей поверхности микропирамиды показан на вставке к рис. 2, $a$. На спектре видны составляющие, соответствующие: $(i)$ люминесценции квантовой ямы InGaN (большая ширина пика объясняется возмущением всей грани микропирамиды, содержащей области с разным составом); (ii) люминесценции слоя $n-\mathrm{InGaN}$, расположенного в ядре микропирамиды; (iii) люминесценции, соответствующей по энергии кванта величине запрещенной зоны $\mathrm{GaN}$.

Ранее показано [21], что при последовательном увеличении тока накачки инжекция в первую очередь происходит в области, соответствующие высокому составу по индию квантовой ямы, а потом уже распространяется в области с меньшим содержанием индия. Анализ спектров и карт КЛ показывает аналогичное поведение исследованных образцов: с увеличением уровня накачки в первую очередь накачивается область вблизи вершины микропирамиды, соответствующая высокому составу по индию квантовой ямы. С повышением уровня накачки наблюдается дополнительный вклад в спектр ЭЛ, обусловленный вкладом области вблизи основания микропирамиды. В то же время при дальнейшем повышении тока накачки появляется пик в спектре ЭЛ на длине волны $400 \mathrm{~nm}$. Данный коротковолновый пик 
возникает вследствие нежелательной накачки слоя, расположенного вне активной области (overshooting effect) и соответствует ЭЛ $n$-InGaN ядра микропирамиды. При максимальном токе накачки также появляется дополнительный пик, соответствующий энергии кванта $3.41 \mathrm{eV}$ (энергия запрещенной зоны $\mathrm{GaN}$ ), возникающий по причине накачки в область $p$ - $\mathrm{GaN}$ оболочки.

Сопоставление изображений карт ЭЛ с изображениями, полученными методом СЭМ, подтверждающие факт ЭЛ одиночной микропирамиды, представлено на рис. 3,c-e. Дополнительным фактом, свидетельствующим об ЭЛ одиночной микропирамиды, является сохранение площади ЭЛ с увеличением токовой накачки. Согласно рис. 3, $a$, FWHМ пика ЭЛ в зеленой области спектра излучения составляет $0.16 \mathrm{eV}$. Также наряду с исследованиями ЭЛ одиночных микропирамид проведены измерения спектров ЭЛ массива, состоящего из малого количества микропирамид, при различных уровнях накачки (рис. $3, b)$. Вставка демонстрирует визуализацию ЭЛ от 5 микропирамид. Спектры ЭЛ демонстрируют те же составляющие, что и спектры ЭЛ одиночных микропирамид (рис. 3,a). FWHМ основного пика спектра ЭЛ с изменением уровня накачки возрастает с 0.16 до $0.3 \mathrm{eV}$. Следует отметить, что величина FWHM от массива микропирамид при высоком уровне накачки превышает значения FWHM для случая исследования ЭЛ от одиночной микропирамиды. Как было показано при изучении карт КЛ, основной вклад в уширение дает неоднородность состава квантовых ям (градиент состава по индию в квантовых ямах) в индивидуальных микропирамидах, а не флуктуация состава квантовых ям между различными микропирамидами.

\section{Заключение}

В ходе выполнения работы проведена отработка условий формирования токовой апертуры, сформированы светодиодные гетероструктуры на основе одиночных $\mathrm{InGaN} / \mathrm{GaN}$ микропирамид в геометрии ядро-оболочка. Продемонстрирована ЭЛ (соответствует размеру токовой апертуры $\geq 10 \mu \mathrm{m}$ ) на длине волны излучения 520-590 nm, сдвигающаяся в коротковолновую область спектра с увеличением токовой накачки. Дальнейшие исследования свойств данных микропирамид позволят реализовать точечные сверхкомпактные источники видимого света высокой яркости, представляющие интерес в качестве фотонных зондов высокого разрешения.

Д.В. Денисов благодарит за частичную поддержку исследований Министерство образования и науки РФ (государственное задание № 16.9789.2017/БЧ). Авторы благодарят О. Крылюк за предоставленные образцы микропирамид

\section{Список литературы}

[1] Kim T.-i., McCall J.G., Jung Y.H., Huang X., Siuda E.R., Li Y., Song J., Song Y.M., Pao H.A., Kim R.-H., Lu C., Lee S.D., Song I.-S., Shin G., Al-Hasani R., Kim S., Tan M.P., Huang Y., Omenetto F.G., Rogers J.A., Bruchas M.R. // Science. 2013. V. 340. P. 211-216. doi 10.1126/science. 1232437

[2] Koester R., Hwang J.-S., Salomon D., Chen X., Bougerol C, Barnes J.-P., Dang D.L.S., Rigutti L. Bugallo A.L., Jacopin G., Tchernycheva M., Durand C., Eymery J. // Nano Lett. 2011. V. 11. P. 4839-4845. doi 10.1021/n1202686n

[3] Bi Z., Gustafsson A., Lenrick F., Lindgren D., Hultin O., Wallenberg L.R., Ohlsson B.J., Monemar B., Samuelson L. // J. Appl. Phys. 2018. V. 123. P. 025102. doi 10.1063/1.5010237

[4] Martensson T., Svensson C.P.T., Wacaser B.A., Larsson M.W., Seifert W., Deppert K., Gustafsson A., Wallenberg L.R., Samuelson L. // Nano Lett. 2004. V. 4. P. 1987-1990. doi 10.1021/n10487267

[5] Largeau L., Dheeraj D.L., Tchernycheva M., Cirlin G.E., Harmand J.C. // Nanotechnology. 2008. V. 19. P. 155704. doi 10.1088/0957-4484/19/15/155704

[6] Calarco R., Meijers R.J., Debnath R.K., Stoica T., Sutter E., Lüth H. // Nano Lett. 2007. V. 7. P. 2248-2251. doi $10.1021 / \mathrm{n} 10707398$

[7] Kumaresan V., Largeau L., Madouri A., Glas F., Zhang H., Oehler F., Cavanna A., Babichev A., Travers L., Gogneau N., Tchernycheva M., Harmand J.-C. // Nano Lett. 2016. V. 16. P. 4895-4902. doi 10.1021/acs.nanolett.6b01453

[8] Kumaresan V., Largeau L., Madouri A., Glas F., Zhang H., Oehler F., Cavanna A., Babichev A., Travers L., Gogneau N., Tchernycheva M., Harmand J.-C. // European Microscopy Congress 2016: Proceedings. 2016. P. 668-669. doi 10.1002/9783527808465.emc2016.6807

[9] Fernández-Garrido S., Ramsteiner M., Gao G., Galves L.A., Sharma B., Corfdir P., Calabrese G., de Souza Schiaber Z., Pfüller C., Trampert A., Lopes J.M.J., Brandt O., Geelhaar L. // Nano Lett. 2017. V. 17. P. 5213-5221. doi 10.1021/acs.nanolett.7b01196

[10] Fernández-Garrido S., Ramsteiner M., Gao G., Galves L.A., Sharma B., Corfdir P., Calabrese G., de Souza Schiaber Z., Pfüller C., Trampert A., Lopes J.M.J., Brandt O., Geelhaar L. // Proc. SPIE. 2018. V. 10532. P. 105321U. doi 10.1117/12.2288233

[11] Guan N., Dai X., Babichev A.V., Julien F.H., Tchernycheva $M$. // Chem. Sci. 2017. V. 8. P. 7904-7911. doi $10.1039 / \mathrm{c} 7 \mathrm{sc} 02573 \mathrm{~d}$

[12] Tian Z., Li Y., Su X., Feng L., Wang S., Ding W., Li Q., Zhang Y., Guo M., Yun F., Lee S.W.R. // Opt. Express. 2018. V. 26. P. 1817. doi 10.1364/oe.26.001817

[13] Choi J.H., Cho E.H., Lee Y.S., Shim M.-B., Ahn H.Y., Baik C.W., Lee E.H., Kim K., Kim T.H., Kim S., Cho K.-S., Yoon J., Kim M., Hwang S. // Adv. Opt. Mater. 2013. V. 2. P. 267-274. doi 10.1002/adom.201300435

[14] Kato Y., Kitamura S., Hiramatsu K., Sawaki N. // J. Cryst. Growth. 1994. V. 144. P. 133-140. doi 10.1016/00220248(94)90448-0

[15] Kitamura S., Hiramatsu K., Sawaki N. // Jpn. J. Appl. Phys. 1995. V. 34. P. L1184-L1186. doi 10.1143/jjap.34.11184

[16] Bidnyk S., Little B.D., Cho Y.H., Krasinski J., Song J.J., Yang W., McPherson S.A. // Appl. Phys. Lett. 1998. V. 73. P. 2242-2244. doi 10.1063/1.121689 
[17] Ko Y.-H., Kim J.-H., Jin L.-H., Ko S.-M., Kwon B.-J., Kim J., Kim T., Cho Y.-H. // Adv. Mater. 2011. V. 23. P. 5364-5369. doi 10.1002/adma.201102534

[18] Stankevic T., Mickevicius S., Schou Nielsen M., Kryliouk O., Ciechonski R., Vescovi G., Kryliouk O., Ciechonski R., Vescovi G., Bi Z., Mikkelsen A., Samuelson L., Gundlach C., Feidenhans'l R. // J. Appl. Crystallogr. 2015. V. 48. P. 344-349. doi 10.1107/s1600576715000965

[19] Song D.Y., Chandolu A., Stojanovic N., Nikishin S.A., Holtz M. // J. Appl. Phys. 2008. V. 104. P. 064309. doi $10.1063 / 1.2978382$

[20] Tong G., Jia W., Fan T., Dong H., Li T., Jia Z., Xu B. // Mater. Lett. 2018. V. 224. P. 86-88. doi 10.1016/j.matlet.2018.04.089

[21] Tchernycheva M., Lavenus P., Zhang H., Babichev A.V., Jacopin G., Shahmohammadi M., Julien F.H., Ciechonski R., Vescovi G., Kryliouk O. // Nano Lett. 2014. V. 14. P. 2456-2465. doi 10.1021/n15001295

[22] Stankevič T., Hilner E., Seiboth F., Ciechonski R., Vescovi G., Kryliouk O., Johansson U., Samuelson L., Wellenreuther G., Falkenberg G., Feidenhans'l R., Mikkelsen A. // ACS Nano 2015. V. 9. P. 6978-6984. doi 10.1021/acsnano.5b01291

[23] Kempa T.J., Lieber C.M. // Pure Appl. Chem. 2014. V. 86. P. 13-26. doi 10.1515/pac-2014-5010

[24] Tchernycheva M., Neplokh V., Zhang H., Lavenus P., Rigutti L., Bayle F., Julien F.H., Babichev A., Jacopin G., Largeau L., Ciechonski R., Vescovi G., Kryliouk O. // Nanoscale. 2015. V. 7. P. 11692-11701. doi $10.1039 / \mathrm{c} 5 \mathrm{nr} 00623 \mathrm{f}$

[25] Hultin O., Otnes G., Borgström M.T., Björk M., Samuelson L., Storm K. // Nano Lett. 2015. V. 16. P. 205-211. doi 10.1021/acs.nanolett.5b03496

[26] Thompson A.C., Stoddart P.R., Jansen E.D. // Curr. Mol. Imaging. 2015. V. 3. P. 162-177. doi 10.2174/2211555203666141117220611

[27] Goßler C., Bierbrauer C., Moser R., Kunzer M., Holc K., Pletschen W., Köhler K., Wagner J., Schwaerzle M., Ruther P., Paul O., Neef J., Keppeler D., Hoch G., Moser T., Schwarz U.T. // J. Phys. D: Appl. Phys. 2014. V. 47. P. 205401. doi 10.1088/0022-3727/47/20/205401

[28] Ayub S., Gossler C., Schwaerzle M., Klein E., Paul O., Schwarz U.T., Ruther P. // IEEE 29th Int. Conf. MEMS. 2016. P. 379. doi 10.1109/memsys.2016.7421640

[29] Soltan A., McGovern B., Drakakis E., Neil M., Maaskant P., Akhter M., Lee J.S., Degenaar P. // IEEE Trans. Biomed. Circuits Syst. 2017. V. 11. P. 347-359. doi 10.1109/tbcas.2016.2623949

[30] Metzner S., Bertram F., Karbaum C., Hempel T., Wunderer T., Schwaiger S., Lipski F., Scholz F., Wächter C., Jetter M., Michler P., Christen J. // Phys. Stat. Solidi B. 2011. V. 248. P. 632-637. doi 10.1002/pssb.201046500 